Article

\title{
Emergence and Spread of SARS-CoV-2 Lineages B.1.1.7 and P.1 in Italy
}

\author{
Francesca Di Giallonardo ${ }^{1, *(1)}$, Ilaria Puglia ${ }^{2}$, Valentina Curini ${ }^{2}(0)$, Cesare Cammà ${ }^{2}\left(\mathbb{D}\right.$, Iolanda Mangone $^{2}$, \\ Paolo Calistri $^{2} \mathbb{D}$, Joanna C. A. Cobbin ${ }^{3}$, Edward C. Holmes ${ }^{3} \mathbb{D}$ and Alessio Lorusso ${ }^{2} \mathbb{D}$ \\ 1 The Kirby Institute, UNSW Sydney, Sydney, NSW 2052, Australia \\ 2 Istituto Zooprofilattico Sperimentale dell'Abruzzo e del Molise G. Caporale, 641000 Teramo, Italy; \\ i.puglia@izs.it (I.P.); v.curini@izs.it (V.C.); c.camma@izs.it (C.C.); i.mangone@izs.it (I.M.); p.calistri@izs.it (P.C.); \\ a.lorusso@izs.it (A.L.) \\ 3 Marie Bashir Institute for Infectious Diseases and Biosecurity, School of Life \& Environmental Sciences and \\ School of Medical Sciences, The University of Sydney, Sydney, NSW 2006, Australia; \\ joanna.cobbin@sydney.edu.au (J.C.A.C.); edward.holmes@sydney.edu.au (E.C.H.) \\ * Correspondence: fdigiallonardo@kirby.unsw.edu.au; Tel.: +61-2-9385-0465
}

check for updates

Citation: Di Giallonardo, F.; Puglia, I.; Curini, V.; Cammà, C.; Mangone, I.; Calistri, P.; Cobbin, J.C.A.; Holmes, E.C.; Lorusso, A. Emergence and Spread of SARS-CoV-2 Lineages B.1.1.7 and P.1 in Italy. Viruses 2021, 13, 794. https:/ / doi.org/10.3390/v13050794

Academic Editor: Stefano Aquaro

Received: 9 April 2021

Accepted: 26 April 2021

Published: 29 April 2021

Publisher's Note: MDPI stays neutral with regard to jurisdictional claims in published maps and institutional affiliations.

Copyright: (c) 2021 by the authors. Licensee MDPI, Basel, Switzerland. This article is an open access article distributed under the terms and conditions of the Creative Commons Attribution (CC BY) license (https:/ / creativecommons.org/licenses/by/ $4.0 /)$.

\begin{abstract}
Italy's second wave of SARS-CoV-2 has hit hard, with more than three million cases and over 100,000 deaths, representing an almost ten-fold increase in the numbers reported by August 2020. Herein, we present an analysis of 6515 SARS-CoV-2 sequences sampled in Italy between 29 January 2020 and 1 March 2021 and show how different lineages emerged multiple times independently despite lockdown restrictions. Virus lineage B.1.177 became the dominant variant in November 2020, when cases peaked at 40,000 a day, but since January 2021 this is being replaced by the B.1.1.7 'variant of concern'. In addition, we report a sudden increase in another documented variant of concern-lineage P.1-from December 2020 onwards, most likely caused by a single introduction into Italy. We again highlight how international importations drive the emergence of new lineages and that genome sequencing should remain a top priority for ongoing surveillance in Italy.
\end{abstract}

Keywords: SARS-Cov-2; Italy; epidemic; variant of concern; B.1.1.7; P.1

\section{Introduction}

COVID-19, caused by infection with severe respiratory coronavirus 2 (SARS-CoV-2), has had a devastating global impact, with an estimated 116 million cases and 2.5 million deaths [1]. Of these reported infections, more than $78 \%$ have occurred between September 2020-March 2021, greatly outnumbering the 25 million cases and $\sim 800 \mathrm{k}$ deaths reported globally prior to 31 August 2020 [2]. This second wave of infections is also characterized by the emergence of numerous new lineages of SARS-CoV-2, three of which are commonly referred to global 'variants of concern'-B.1.1.7, B.1.351, and P.1 [3]. The B.1.1.7 lineage was first detected in the United Kingdom in late September 2020 [4] and is characterized by 17 changes across the genome including the N501Y amino acid mutation and a two amino acid deletion at positions 69 and 70 of the spike protein [5]. The B.1.351 lineage was first reported in South Africa and shares similar substitutions to B.1.1.7 including N501Y but not the 69/70 deletion [6], while the P.1 lineage was first identified in Brazil and shares two substitutions with B.1.351-E484K and N501Y in the spike protein [4]. All of these lineages have seemingly replaced previous circulating variants in their geographic regions and have spread to other countries in Europe, the Americas, and Asia [7-9]. There is also considerable concern about the possibility of reinfection with these new lineages due to reduced cross-protective immunity [10-12], which may also have implications for vaccine efficacy $[13,14]$. Thus, increased surveillance to document and understand the spread of different SARS-CoV-2 lineages is of upmost importance.

The first cases of SARS-CoV-2 in Italy were reported in January 2020 and the country was hit hard with a peak of $>6500$ cases a day in late March 2020. Italy was also the first European 
country to impose a nation-wide lockdown from 9 March to the 18 May 2020. In retrospect, this first wave was of relatively low magnitude compared to the huge number of infections reported between September 2020 and March 2021. Indeed, during this second wave, Italy experienced a maximum of $\sim 40,000$ cases a day in mid-November 2020 (Figure 1a), and an estimated 3.1 million cases and $~ 100,000$ deaths have been reported to date (8 March 2021) [15]. Beginning in October 2020, a color-coded system was established to restrict the mobility of residents in each administrative region in Italy, depicting increasing the levels of restriction from yellow to red. In addition, in attempt to reduce viral transmission during the holiday season, further restrictions to social mobility and on possible sources of infection (schools, restaurants, etc.) were put in place for the whole of Italy between 21 December 2020 and 6 January 2021. On 20 December 2020, the Italian Minister of Health signed an order prohibiting all air flights from/to United Kingdom. On 16 January 2021 the same restriction was applied to the air flight from/to Brazil.

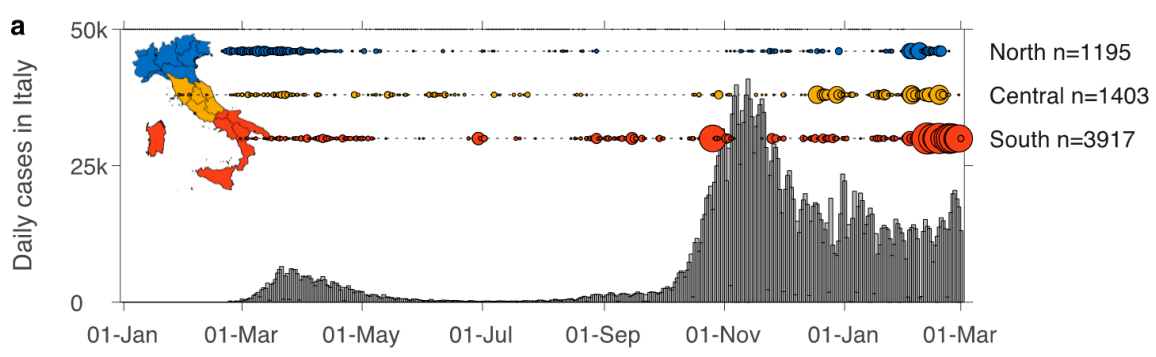

b
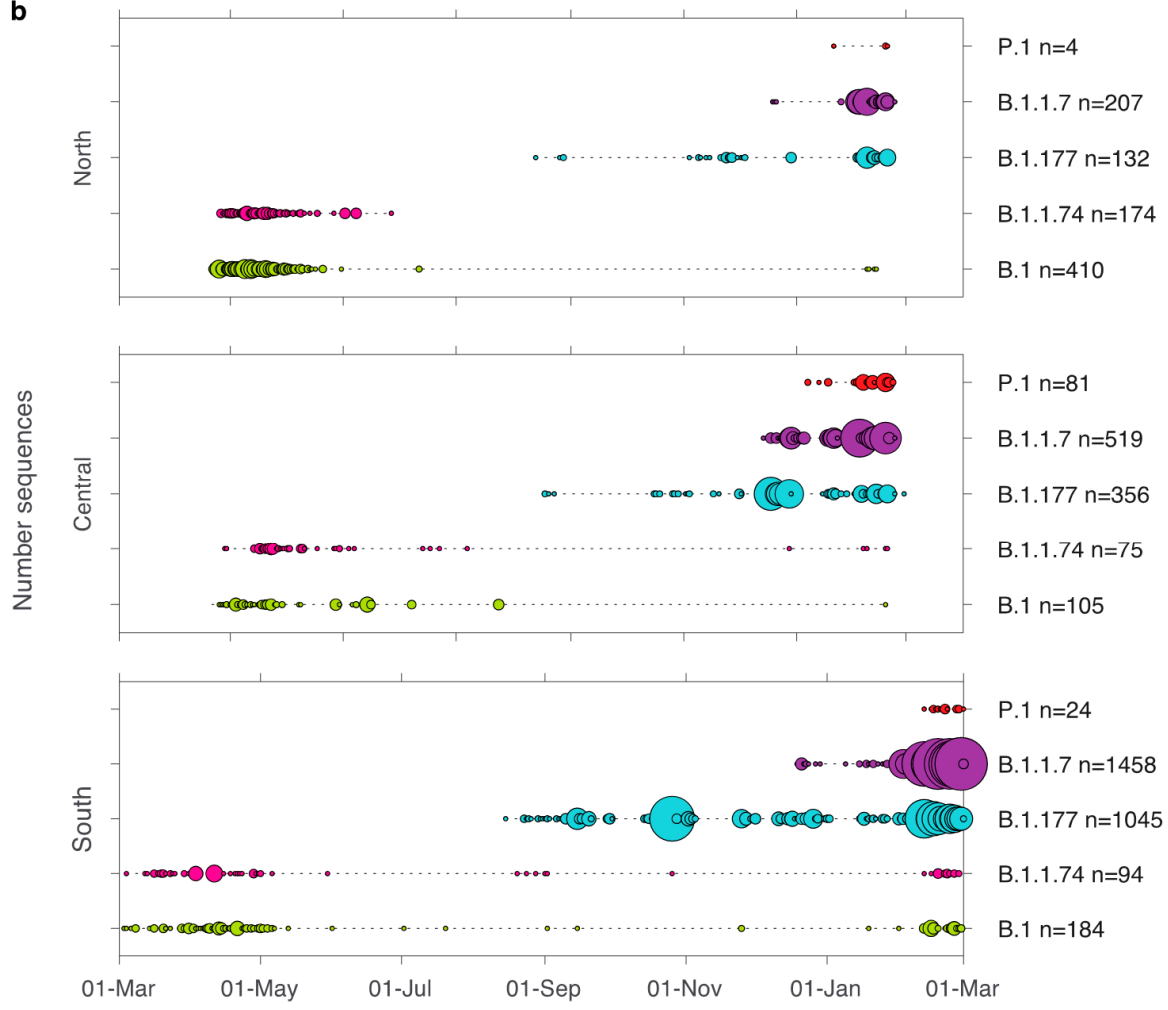

Figure 1. Number of SARS-CoV-2 genomes sampled over time in Italy. (a) Bars show the number of cases reported in Italy between 1 January 2020-1 March 2021. Horizontally aligned circles show the number of sequences sampled for each day in North (blue), Central (yellow), South (red) Italy. The size of the circle is equivalent to the total number of sequences $(\max =256)$. Italian regions are coloured according to their macro areas: blue = North (Valle d'Aosta, Piemonte, Liguria, Lombardia, Emilia-Romagna, Veneto, Friuli-Venezia-Giulia, and Trentino-Alto Adige), yellow = Central (Lazio, Marche, Toscana, Umbria, and Abruzzo), red = South (Puglia, Basilicata, Calabria, Campania, Molise, Sicilia, and Sardegna). (b) Number of sequences sampled in Italy for the lineages B.1. B.1.1.74, B.1.177, B.1.1.7, and P.1 for North (top), Central (middle), and South (bottom) Italy. The total number of sequences per day (circles) and the time range between first and most recent sequence (dotted line) is shown ( $\max =146)$. 
We previously showed that virus importation associated with travel, followed by local transmission, were key drivers of viral spread during the first wave of SARS-CoV-2 in Italy [16]. After a year into the pandemic, lockdown restrictions have proven effective in reducing virus transmission and limiting geographic spread [17], although international travel is still a major source for the introduction of new lineages into Italy [18]. Here we show how the B.1.1.7 and P.1 variants entered Italy before the implementation of border closures and that these two variants have subsequently spread rapidly across the country.

\section{Materials and Methods}

\subsection{Sequence Data}

In Italy, the Department of Health Prevention, itself organized into nearly 100 Local Health Units (ASL), are also in charge of implementing all SARS-CoV-2 surveillance and tracing activities. Surveillance is mainly based on nasopharyngeal swab testing by real time RT-PCR in approved public laboratories, and performed on all individuals with clinical signs compatible with COVID-19 (persisting fever, dyspnea, anosmia, etc.) as well as those in strict contact with confirmed cases in the 14 days before clinical onset. In addition, mass drive-through testing initiatives that make use of antigenic rapid tests are organized in ports, airports, main train and bus stations, major cities, and towns experiencing a significant increase in case numbers. People with positive results to antigenic rapid tests are then subjected to a follow-up swab for molecular testing. In addition, periodic testing is performed on at-risk worker categories, such as health and laboratory personnel. Sequencing and genome analysis activities based on direct infected swab samples are still far from optimal, and are performed on a voluntary basis by equipped laboratories scattered all over the Italian territory. However, of a total of 834,274 sequences available on the GISAID database (https:/ / www.gisaid.org/ (accessed on 22 March 2021)) up to 22 March 2021, only 13,769 originated from Italy, with huge differences between regions.

All Italian sequences of SARS-CoV-2 available on the GISAID database were downloaded (8 March 2021, Supplementary Table S1). Sequences without a complete date of collection were removed. As per our previous study, cases were grouped according to geographical non-administrative macro areas in North $(n=1195)$, Central $(n=1403)$, and South Italy $(n=3917)$ [16]. SARS-CoV-2 lineages were assigned to each sequence using the Pangolin COVID-19 Lineage Assigner tool v2.0.7 (github.com/cov-lineages/pangolin). All available global sequence data for B.1.351 and P.1 were similarly downloaded from the GISAID database. Due to the large amount of data available for B.1.1.7 $(>170,000)$, a random subset of 3000 global sequence data was used in combination with all available Italian sequence data. In brief, a global phylogeny containing 289,000 sequences was downloaded from GISAID [19]. Sequences classifying as B.1.1.7 lineage $(\sim 94,000)$ were extracted from which a random subset of 3000 sequences was selected. A list with accession numbers for the global sequence data used in the phylogenies can be found in Supplementary Table S2.

\subsection{Phylogenetic Analysis}

Separate nucleotide sequence alignments were constructed for the B.1.1.7, P.1, and B.1.351 lineages. A representative sequence for each lineage was used as a reference and as an outgroup for tree rooting (B.1.1.7 = EPI_ISL_862239, B.1.351 = EPI_ISL_660190, P.1 = EPI_ISL_833137). Three separate alignments were performed using MAFFT implementing the L-INS-I algorithm and manually inspected for accuracy using Geneious Prime ${ }^{\circledR}$ 2021.1.1 (https: / / www.geneious.com (accessed on 12 March 2021)) [20]. Full genome sequences with $>5 \%$ ambiguity and no exact sampling date were removed. The final data sets comprised: P.1 $n=614$ (Italy $n=111$ ), B.1.351 $n=2317$ (Italy $n=8$ ), and B.1.1.7 $n=4361$ (Italy $n=1461$ ). A maximum likelihood tree was estimated for each lineage using IQTREE implementing the Hasegawa-Kishino-Yano nucleotide substitution model with a gamma distributed rate variation among sites $(\mathrm{HKY}+\Gamma)$ and an ultrafast bootstrap method (1000 repetitions) [21,22]. 


\section{Results}

\subsection{Emerging Lineages in Italy}

Our analysis included 6515 Italian SARS-CoV-2 sequences covering a time span between 29 January 2020 to 1 March 2021. These comprised 103 different lineages, with B.1 (11\%), B.1.1.74 (5\%), B.1.1.7 (34\%), and B.1.177 (24\%) the most commonly identified. Sequence sampling was not equal between North, Central, and South Italy, as a large proportion of SARS-CoV-2 sequence data from North Italy was only available for the beginning of the pandemic (March-May 2020) and again the most recent months (JanuarMarch 2021) (Figure 1a) Despite this, the time range of data availability overlaps between these three macro areas, minimizing the effect of sampling bias.

Lineages B.1 and B.1.1.74 both appeared in Italy during early 2020 and were replaced by lineage B.1.177 during the second half of 2020 (Figure 1b). Lineage B.1 was the first reported in all three macro areas on 20 February, 24 February, and 3 March in North, Central, and South Italy, respectively. A similarly narrow time period of introduction was observed for lineage B.1.1.74 which first appeared on 24 February, 27 February, 4 March 2020 in North, Central, and South Italy, respectively. This latter lineage persisted in Central and South Italy until February 2021, although at low levels, while no sequences from this lineage were sampled in North Italy after 27 May 2020. Subsequently, lineage B.1.177 emerged in August in all three macro areas becoming the dominant variant in the second wave, with the earliest documentation in North Italy (13 August), followed by South Italy on 15 August and Central Italy on 18 August 18.

Although these data show that different lineages of SARS-CoV-2 were first reported within a short time period throughout the country, we also identified region-specific sampling biases with unusual abundances of sequences obtained on individual days (Supplementary Figure S1). These sampling 'peaks' are most likely the result of targeted sampling after an outbreak. For example, 193 sequences were sampled on 26 October 2020 alone, of which 104 were B.1.177 all sampled in the region Campania in South Italy. Also, in this region, 42 B.1.1.187 sequences were sampled on 29 June 2020 alone, and which represents $51 \%$ of all sequence data available for this lineage in Italy. Notably, according to GISAID, this lineage has only been sampled in the UK $(n=14)$ and Japan $(n=1)$, and South Italy $(n=81)$.

\subsection{Lineages of Global Concern-B.1.1.7, P.1, and B.1.351}

At the time of writing (8 March 2021), a total of 2184 B.1.1.7, 109 P.1, and 8 B.1.351 sequences sampled in Italy were available for analysis and had been identified in all three Italian macro areas. The B.1.1.7 lineage appeared in all three areas within a five-day period, first in Central Italy (14 December) and five days later in both the North and South (19 December for both lineages) (Figure 1b). The first P.1 sequences were identified on 7 January 2021 in Central Italy in travelers returning from Brazil [23]. This lineage was subsequently found on 21 January and 12 February in North and South Italy, respectively.

Notably, the Italian B.1.1.7 sequences were distributed across the global phylogeny indicating multiple independent introductions into the country (Figure 2). In addition, we observed numerous Italian-specific transmission clades (i.e., extended transmission chains), although most ( $n=73,78 \%$ ) were area specific, the largest containing 104 infections all sampled in South Italy (Figure 2). Of the remaining clades $(n=22), 82 \%$ contained infections from two and 18\% from all three macro areas. For $73 \%(n=16)$ of these, the clade in question comprised $\geq 80 \%$ of infections from a single area only and only a small number of infections from a second or third macro area. For example, one clade with 64 sequence comprised 63 from Central Italy, one from South Italy and none from North Italy. Similarly, another clade contained 76 infections from North Italy and one from Central Italy. In addition, we identified six clades with more inter-area mixing, including one example consisting of 35 infections (10 North, 12 Central, 13 South). Notably, this is the only example of prolonged transmission between macro areas for this time period (Figure 2). 


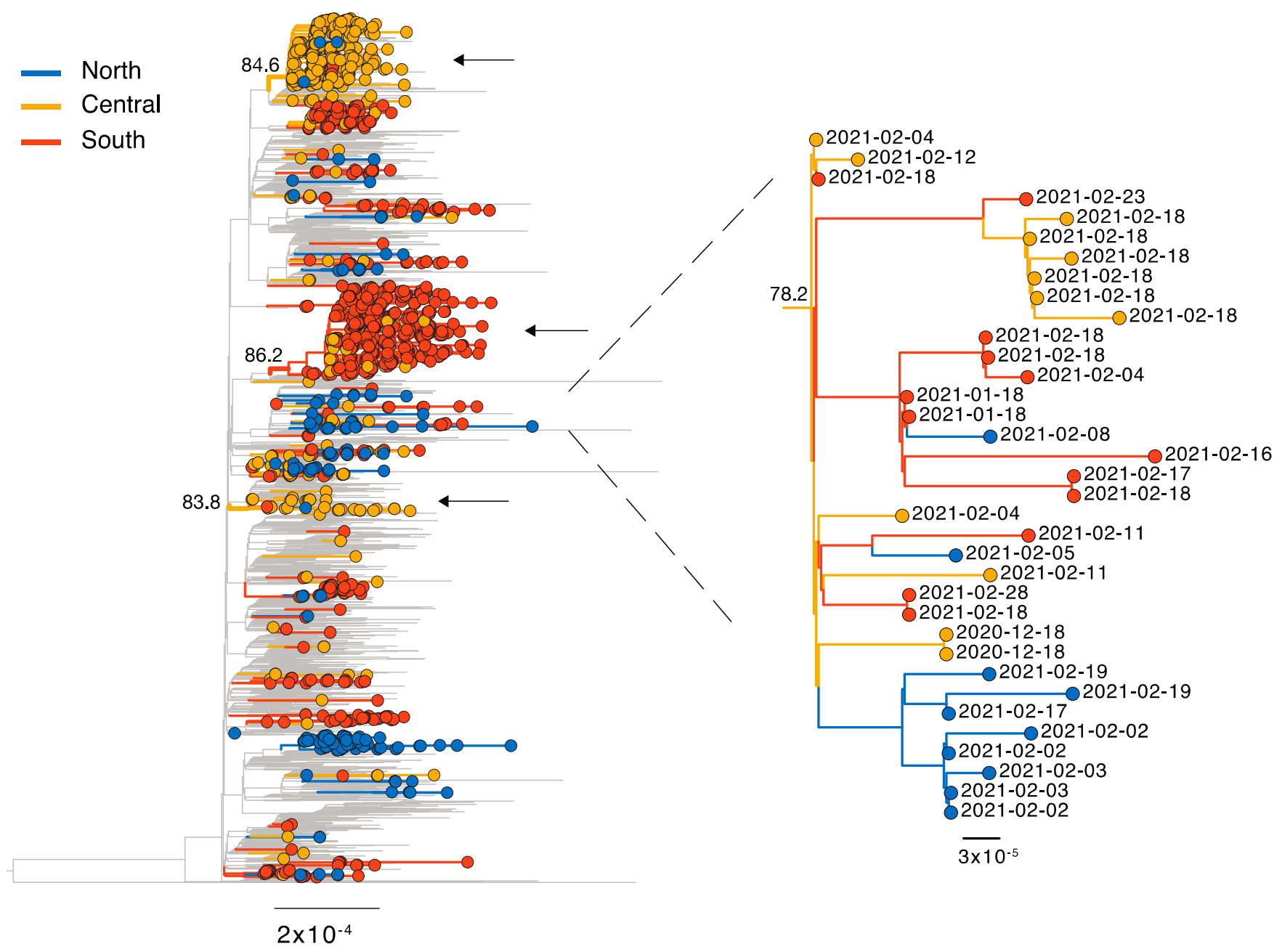

Figure 2. Genomic epidemiology of SARS-CoV-2 lineage B.1.1.7. Maximum likelihood phylogeny of SARS-CoV-2 full genome sequences. Due to the large amount of data for B.1.1.7 (>100,000 sequences), a random subset of 3000 B.1.1.7 sequences sampled globally were incorporated (2900 after data cleaning). Branches are colored according to the macro area: grey $=$ global, blue $=$ North Italy, yellow $=$ Central Italy, red $=$ South Italy. Branch length is scaled according to the number of nucleotide substitutions per site. Lineage B.1.351 and P.1 were used as an outgroup (B.1.351 = EPI_ISL_660190, P.1 = EPI_ISL_833137). Arrows indicate examples of large Italian clades with limited inter-region transmission; bootstrap node support is indicated. One clade with increased inter-region transmission is shown enlarged on the right.

Three infections of the P.1 lineage were identified in international travelers on 18 January in Central Italy. Fortunately, these did not lead to ongoing local transmission (Figure 3). However, infections of P.1 were also identified on 7 January, and which formed a distinct clade (clade I) within the P.1 global phylogeny distinguished by a single T- > C synonymous mutation in ORF1ab (nt 13,577/aa 4526 Ref EPI_ISL_833137). In contrast to B.1.1.7, almost all of Italian P.1 sequences $(n=104)$ fell within this single node which also contained 10 sequences from Germany (Figure 3). Within this clade I, 61 Italian sequences (clade II) contained an additional distinct synonymous mutation in the spike gene (spike nt 3357 / aa 1118 Ref EPI_ISL_833137), and 43 of these Italian sequences (clade III) also contained a non-synonymous mutation in the spike leading to an S813N mutation (spike nt 2437/aa 813 Ref EPI_ISL_833137). This S813N mutation is located between two fusion peptides and not in the receptor binding domain where the E484K and N501Y mutations are located (Supplementary Figure S2). P.1. viruses with the mutation were first sampled on 13 January 2021 in Central Italy and the most recent occurrence was found in an infection sampled on 1 March. To date, the mutation has only been found in infections from Central and South Italy. Of note, one additional sequence (EPI_ISL_1169907, Central Italy, 
19-February) outside of both clade II and III also contained this amino acid substitution (Figure 3).

a

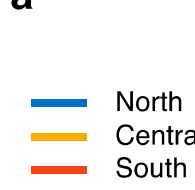

b

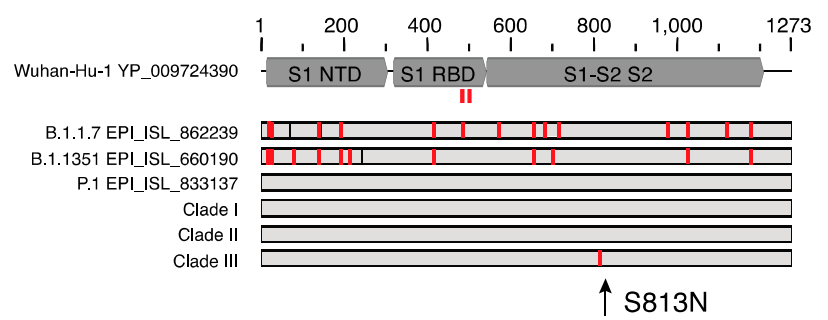

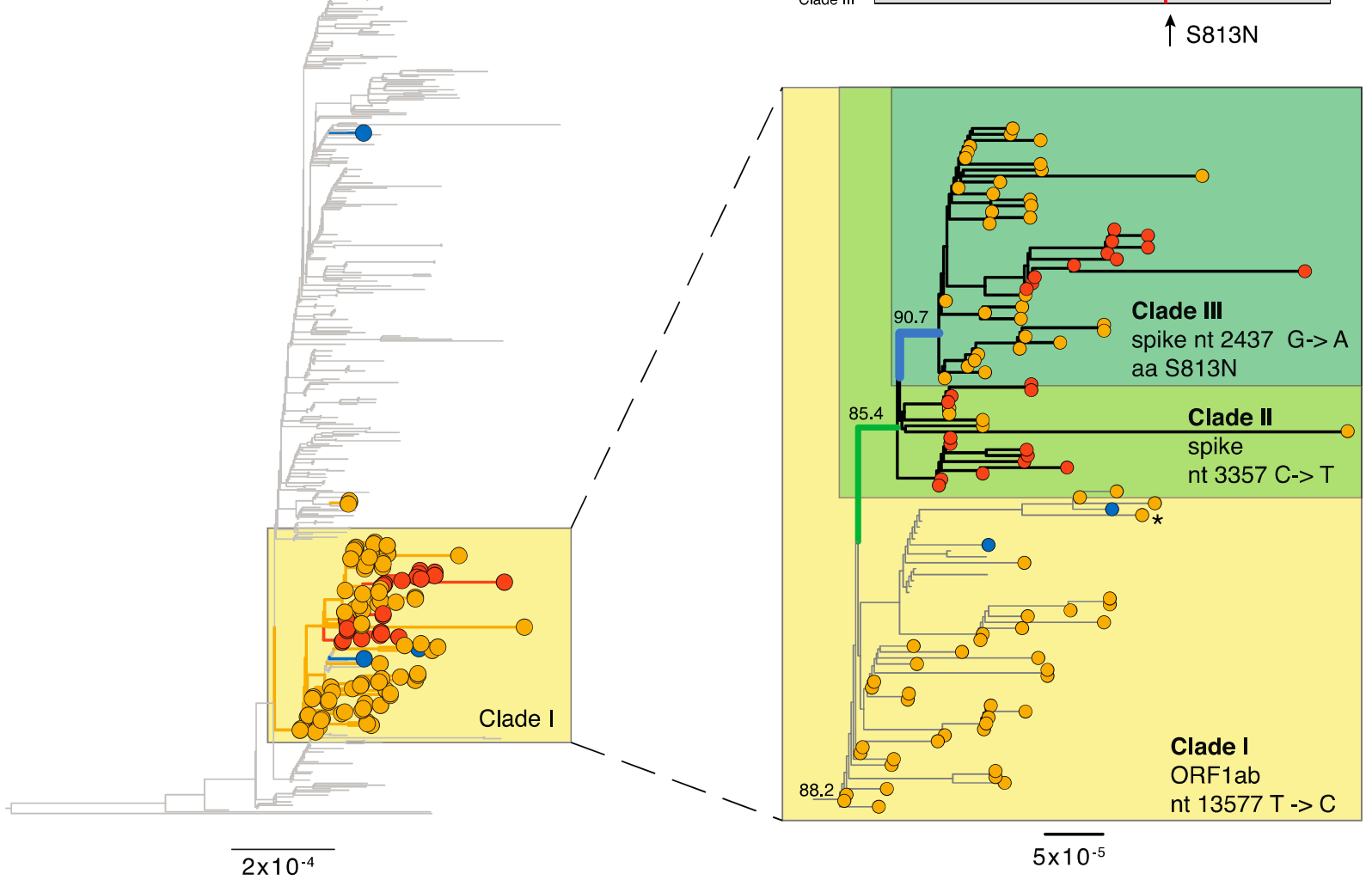

Figure 3. Genomic epidemiology of SARS-CoV-2 lineage P.1. (a) Maximum likelihood phylogeny of SARS-CoV-2 full genome sequences ( $n=503$ global, $n=111$ Italian). Lineage B.1.351 was used as an outgroup (EPI_ISL_660190). Branch lengths are scaled according to the number of nucleotide substitutions per site and are colored according to geographic macro area: grey $=$ global, blue $=$ North Italy, yellow $=$ Central Italy, red $=$ South Italy. The node containing the $\mathrm{T}$ to $\mathrm{C}$ mutation in ORF1ab is marked with a yellow box (clade I). (b) (bottom) Enlargement of this node and additional clades and their nucleotide or amino acid substitutions are indicated. Bootstrap node support for clades I-III is shown. Note, a single sequence containing also the $5813 \mathrm{~N}$ mutation but falling in clade I is marked with an asterisk. Branches without a tip circle represent sequences from Germany. (top) Schematic view of the spike protein alignment for different reference sequences as well as the clade containing the S813N substitution. The protein structural regions are marked: S1 NTD = N-terminal domain of the S1 subunit, S1 RBD = receptor binding domain of S1, S1-S2 S2 = S1/S2 cleavage region and S2 fusion subunit. A detailed view of the protein alignment can be found in Supplementary Figure S2.

Finally, five of the eight B.1.351 sequences were scattered across the global phylogeny, confirming that these are independent infections associated with returned travelers (30 January and 11 February in North Italy $n=2,23$ February in Central Italy $n=1,19$ and 26 February in South Italy $n=2$, Supplementary Figure S3). However, we also found B.1.351 sequences from North Italy sampled on 11 and 13 February 2021 who formed a phylogenetic cluster, suggestive of some local transmission. 


\section{Discussion}

We document the complex patterns of virus transmission and lineage turnover during the second wave of SARS-CoV-2 in Italy, with an analysis based on $>6500$ sequences sampled from January 2020-March 2021. First, we observed the replacement of B.1 and B.1.1.74 by B.1.177, the latter being the most sampled lineage during the second wave. Our data shows how different lineages appeared almost simultaneously in North, Central, and South Italy (i.e., within a five-day time frame), suggesting a complex pattern of multiple introductions into the different macro areas. Second, and more notably, we observed the more recent appearance of the emerging 'variants of concern' B.1.1.7 and P.1, with the former being the most sampled lineage during January and February 2021.

We focused our analysis on lineages B.1.1.7, B.1.351, and P.1 as these have been the subject of considerable discussion and concern. An importation of B.1.351 was first reported by Novazzi et al. [24], with this variant identified in a returning traveler from Malawi, although this did not lead to ongoing transmission. Indeed, in the case of B.1.351, we only found one instance of local transmission in Italy: three infections from mid-February 2021, with no evidence for additional ongoing transmission. In contrast, we found strong evidence for local ongoing transmission of both the B.1.1.7 and P.1 lineages, with the former potentially replacing the previous dominant strain B.1.177. Of note, from 8 March 2021 onwards, B.1.1.7 represented on average $50 \%$ of all sequences sampled. This is compatible with observations that B.1.1.7 has enhanced transmissibility [5] and has become the dominant variant in numerous other European countries, e.g., Denmark [25].

Our phylogenetic analysis confirms extensive virus transmission for B.1.1.7 within North, Central, and South Italy, but only limited transmission between these regions, consistent with lockdown restrictions imposed in December 2020 that prohibited travel between regions. We did find one cluster containing a mix of sequences sampled from infection in North, Central, and South Italy. However, the limited sequence data available as well as the lack of metadata makes it challenging to accurately identify the directionality of transmission and lineage spread across the country, although our previous study similarly showed limited interregional transmission during the lockdown period [16]. Although no metadata regarding virulence was available for the sequence data included here, it was recently shown that some B.1.1.7 variants are characterized by longer persistence and higher viral RNA loads in nasopharyngeal swabs [26], in the UK the B.1.1.7 is associated with both a higher production number and a greater hazard of death [5]. Of note, no clade defining amino acid substitutions were found for the B.1.1.7 lineage in spike gene of Italian sequences analyzed here.

After Brazil, Italy harbors the second highest number of P.1 infections reported to date [3]. This variant was first reported in Manaus (Brazil), which was hit hard by the first wave of SARS-CoV-2 with an estimated $76 \%$ of the population infected [27]. Thus, the surge of P.1 in this area strongly implies the possibility of reinfection [9]. An earlier study reported the presence of P.1 in three returning travelers from Brazil to Abruzzo sampled on 18 January 2021 [23], although we show here that this small cluster did not lead to (detectable) subsequent transmission events. A second study reported the presence of P.1 in a returning traveler from Brazil [28]. This individual was sampled on 22 January in Milan, North Italy. However, as the sequence deposited only covered the spike protein, we did not include it in our analysis. Despite this our molecular epidemiological analysis provided strong evidence for a single introduction of P.1 into Italy, followed by extensive local transmission. Indeed, our phylogenetic analysis depicts importation of the P.1 variant into Italy, although from a yet unknown source, followed by possible onward spread from Italy to Germany. Importantly, within the Italian network we also found a unique, clade defining amino acid substitution-S813N. While the function of this mutation is currently unknown, it is likely unrelated to virus binding and hence does not represent an immune escape variant, although this should be assessed further [13,29].

Our study contains notable limitations. Although we included all available sequence data from GISAID ( 6000 sequences), this represents a tiny fraction of the total number 
infections reported in Italy to date ( $>3$ million). We also observed a marked sampling bias between North, Central, and South Italy, which hampers estimates on the extent of interregional transmission. Finally, our study focused on three key variants only and we did not report the transmission patterns for B.1 and B.1.177, which may have greater levels of inter-region transmission following the easing of population lockdowns.

\section{Conclusions}

We depict the rapid emergence and replacement of new lineages of SARS-CoV-2 in Italy despite major lockdown restrictions, suggesting that the disease management policies employed were insufficient to halt the spread of emerging variants. Notably, both the B.1.1.7 and P.1 variants of concern spread across Italy before international border closers were imposed for the UK and Brazil. This highlights the importance of a rapid and inclusive vaccine roll-out on a global scale.

Supplementary Materials: The following are available online at https://www.mdpi.com/article/ 10.3390/v13050794/s1, Figure S1: Changing frequency of SARS-CoV-2 lineages sampled over time in Italy, Figure S2: Amino acid alignment of the SARS-CoV-2 spike protein, Figure S3: Molecular epidemiology of global SARS-CoV-2 lineage B.1.351, Table S1: GISAID acknowledgments, Table S2: List of global SARS-Cov-2 sequence accession numbers.

Author Contributions: Conceptualization F.D.G., E.C.H. and A.L.; methodology and formal analysis, F.D.G. and J.C.A.C.; investigation I.P., V.C., C.C., I.M., P.C., A.L.; resources, A.L.; data curation, A.L.; writing—original draft preparation, F.D.G., E.C.H., and A.L.; visualization, F.D.G.; supervision, E.C.H. and A.L.; project administration, A.L.; funding acquisition, A.L. and E.C.H. All authors have read and agreed to the published version of the manuscript.

Funding: A.L. was funded by the European Union's Horizon 2020 Research and Innovation program under grant agreement No 773830: One Health European Joint Program and by the Italian Ministry of Health IZS AM 08/19 Ricerca Corrente 2019, "NGS e diagnostica molecolare in Sanità Animale: Fast D2" and IZSAM 05/20 Ricerca Corrente 2020 "PanCO: epidemiologia e patogenesi dei coronavirus umani ed animali". E.C.H. was funded by an Australian Research Council Australian Laureate Fellowship (FL17010002).

Institutional Review Board Statement: Not applicable.

Informed Consent Statement: Not applicable.

Data Availability Statement: Sequence data is available via GISAID. The accession numbers for the sequences used can be found in the Supplementary Material.

Acknowledgments: We are grateful to all individuals who submit data to GISAID. We thank the Pango team for their efforts in maintaining a freely available tool for lineage classification. The authors would like to acknowledge all doctors, nurses, technicians, medical staff, administrators, food and cleaning service workers, pharmacists, and all other members of the COVID-19 diagnostic group at IZSAM.

Conflicts of Interest: The authors declare no conflict of interest.

\section{References}

1. WHO. Weekly Operational Update on COVID-19-8 March 2021; WHO: Geneva, Switzerland, 2021.

2. WHO. Weekly Epidemiological Update—31 August 2020; WHO: Geneva, Switzerland, 2020.

3. Rambaut, A.; Holmes, E.C.; O’Toole, A.; Hill, V.; McCrone, J.T.; Ruis, C.; du Plessis, L.; Pybus, O.G. A dynamic nomenclature proposal for SARS-CoV-2 lineages to assist genomic epidemiology. Nat. Microbiol. 2020, 5, 1403-1407. [CrossRef] [PubMed]

4. CDC. Science Brief: Emerging SARS-CoV-2 Variants. Available online: https://www.cdc.gov/coronavirus/2019-ncov/more/ science-and-research/scientific-brief-emerging-variants.html (accessed on 1 March 2021).

5. Davies, N.G.; Abbott, S.; Barnard, R.C.; Jarvis, C.I.; Kucharski, A.J.; Munday, J.D.; Pearson, C.A.B.; Russell, T.W.; Tully, D.C.; Washburne, A.D.; et al. Estimated transmissibility and impact of SARS-CoV-2 lineage B.1.1.7 in England. Science 2021. Online ahead of print. [CrossRef]

6. Tegally, H.; Wilkinson, E.; Giovanetti, M.; Iranzadeh, A.; Fonseca, V.; Giandhari, J.; Doolabh, D.; Pillay, S.; San, E.J; Msomi, N.; et al. Emergence and rapid spread of a new severe acute respiratory syndrome-related coronavirus 2 (SARS-CoV-2) lineage with multiple spike mutations in South Africa. medRxiv 2020. [CrossRef] 
7. O'Toole, A.; Hill, V.; Pybus, O.G.; Watts, A.; Bogoch, I.I.; Khan, K.; Messina, J.P.; consortium, T.C.-G.U.C.-U.; (NGS-SA), N.f.G.S.i.S.A.; Network, B.-U.C.G.; et al. Tracking the International Spread of SARS-CoV-2 lineages B.1.1.7 and B.1.351/501Y-V2. Available online: https:/ / virological.org/t/tracking-the-international-spread-of-sars-cov-2-lineages-b-1-1-7-and-b-1-351-50 1y-v2/592 (accessed on 1 March 2021).

8. Walensky, R.P.; Walke, H.T.; Fauci, A.S. SARS-CoV-2 Variants of Concern in the United States-Challenges and Opportunities. JAMA 2021, 325, 1037-1038. [CrossRef] [PubMed]

9. Sabino, E.C.; Buss, L.F.; Carvalho, M.P.S.; Prete, C.A., Jr.; Crispim, M.A.E.; Fraiji, N.A.; Pereira, R.H.M.; Parag, K.V.; da Silva Peixoto, P.; Kraemer, M.U.G.; et al. Resurgence of COVID-19 in Manaus, Brazil, despite high seroprevalence. Lancet 2021, 397, 452-455. [CrossRef]

10. Resende, P.C.; Bezerra, J.F.; Vasconcelos, R.H.T.d.; Arantes, I.; Appolinario, L.; Mendonça, A.C.; Paixao, A.C.; Rodrigues, A.C.D.; Silva, T.; Rocha, A.S.; et al. Spike E484K Mutation in the First SARS-CoV-2 Reinfection Case Confirmed in Brazil. 2020. Available online: https://virological.org/t/spike-e484k-mutation-in-the-first-sars-cov-2-reinfection-case-confirmed-in-brazil-2020/584 (accessed on 1 March 2021).

11. Naveca, F.; Costa, C.d.; Nascimento, V.; Souza, V.; Corado, A.; Nascimento, F.; Costa, Á.; Duarte, D.; Silva, G.; Mejía, M.; et al. SARS-CoV-2 reinfection by the new Variant of Concern (VOC) P.1 in Amazonas, Brazil. Available online: https://virological.org/ t/sars-cov-2-reinfection-by-the-new-variant-of-concern-voc-p-1-in-amazonas-brazil/596 (accessed on 1 March 2021).

12. Wibmer, C.K.; Ayres, F.; Hermanus, T.; Madzivhandila, M.; Kgagudi, P.; Oosthuysen, B.; Lambson, B.E.; de Oliveira, T.; Vermeulen, M.; van der Berg, K.; et al. SARS-CoV-2 501Y.V2 escapes neutralization by South African COVID-19 donor plasma. Nat. Med. 2021. [CrossRef]

13. Williams, T.C.; Burgers, W.A. SARS-CoV-2 evolution and vaccines: Cause for concern? Lancet Respir. Med. 2021. Online ahead of print. [CrossRef]

14. Xie, X.; Liu, Y.; Liu, J.; Zhang, X.; Zou, J.; Fontes-Garfias, C.R.; Xia, H.; Swanson, K.A.; Cutler, M.; Cooper, D.; et al. Neutralization of SARS-CoV-2 spike 69/70 deletion, E484K and N501Y variants by BNT162b2 vaccine-elicited sera. Nat. Med. 2021. Online ahead of print. [CrossRef] [PubMed]

15. Statistiche Coronavirus. Statistiche Coronavirus in Italia. Available online: https://statistichecoronavirus.it/coronavirus-italia/ (accessed on 8 March 2021).

16. Di Giallonardo, F.; Duchene, S.; Puglia, I.; Curini, V.; Profeta, F.; Camma, C.; Marcacci, M.; Calistri, P.; Holmes, E.C.; Lorusso, A. Genomic epidemiology of the first wave of SARS-CoV-2 in Italy. Viruses 2020, 12, 1438. [CrossRef] [PubMed]

17. Schlosser, F.; Maier, B.F.; Jack, O.; Hinrichs, D.; Zachariae, A.; Brockmann, D. COVID-19 lockdown induces disease-mitigating structural changes in mobility networks. Proc. Natl. Acad. Sci. USA 2020, 117, 32883-32890. [CrossRef] [PubMed]

18. Swadi, T.; Geoghegan, J.L.; Devine, T.; McElnay, C.; Sherwood, J.; Shoemack, P.; Ren, X.; Storey, M.; Jefferies, S.; Smit, E.; et al. Genomic evidence of in-flight transmission of SARS-CoV-2 despite predeparture testing. Emerg. Infect. Dis. 2021, 27, 687-693. [CrossRef] [PubMed]

19. Lanfear, R. A Global Phylogeny of hCoV-19 Sequences from GISAID. Available online: https://www.gisaid.org/ (accessed on 10 March 2020).

20. Kuraku, S.; Zmasek, C.M.; Nishimura, O.; Katoh, K. aLeaves facilitates on-demand exploration of metazoan gene family trees on MAFFT sequence alignment server with enhanced interactivity. Nucleic Acids Res. 2013, 41, W22-W28. [CrossRef] [PubMed]

21. Trifinopoulos, J.; Nguyen, L.T.; von Haeseler, A.; Minh, B.Q. W-IQ-TREE: A fast online phylogenetic tool for maximum likelihood analysis. Nucleic Acids Res. 2016, 44, W232-W235. [CrossRef] [PubMed]

22. Nguyen, L.T.; Schmidt, H.A.; von Haeseler, A.; Minh, B.Q. IQ-TREE: A fast and effective stochastic algorithm for estimating maximum-likelihood phylogenies. Mol. Biol. Evol. 2015, 32, 268-274. [CrossRef] [PubMed]

23. Delli Compagni, E.; Jurisic, L.; Caporale, M.; Baca, F.; Scialabba, S.; Fani, S.; Perullo, A.; Toro, M.; Marchegiano, A.; Martino, M.; et al. Genome sequences of three SARS-CoV-2 P.1 strains identified from patients returning from Brazil to Italy. Microbiol. Resour. Announc. 2021, 10. [CrossRef] [PubMed]

24. Novazzi, F.; Genoni, A.; Spezia, P.G.; Focosi, D.; Zago, C.; Colombo, A.; Cassani, G.; Pasciuta, R.; Tamborini, A.; Rossi, A.; et al Introduction of SARS-CoV-2 variant of concern 20h/501Y.V2 (B.1.351) from Malawi to Italy. Emerg. Microbes Infect 2021, 10, 710-712. [CrossRef] [PubMed]

25. Danish Covid-19 Genome Consortium. Genomic overview of SARS-CoV-2 in Denmark. Available online: https://www.covid1 9genomics.dk/statistics (accessed on 15 March 2021).

26. Calistri, P.; Amato, L.; Puglia, I.; Cito, F.; Di Giuseppe, A.; Danzetta, M.L.; Morelli, D.; Di Domenico, M.; Caporale, M.; Scialabba, S.; et al. Infection sustained by lineage B.1.1.7 of SARS-CoV-2 is characterised by longer persistence and higher viral RNA loads in nasopharyngeal swabs. Int. J. Infect. Dis. 2021, 105, 753-755. [CrossRef] [PubMed]

27. Buss, L.F.; Prete, C.A., Jr.; Abrahim, C.M.M.; Mendrone, A., Jr.; Salomon, T.; de Almeida-Neto, C.; Franca, R.F.O.; Belotti, M.C.; Carvalho, M.; Costa, A.G.; et al. Three-quarters attack rate of SARS-CoV-2 in the Brazilian Amazon during a largely unmitigated epidemic. Science 2021, 371, 288-292. [CrossRef] [PubMed]

28. Maggi, F.; Novazzi, F.; Genoni, A.; Baj, A.; Spezia, P.G.; Focosi, D.; Zago, C.; Colombo, A.; Cassani, G.; Pasciuta, R.; et al. Imported SARS-CoV-2 variant P.1 in traveler returning from Brazil to Italy. Emerg. Infect. Dis. 2021, 27, 1249-1251. [CrossRef] [PubMed]

29. Wise, J. Covid-19: The E484K mutation and the risks it poses. BMJ 2021, 372, n359. [CrossRef] [PubMed] 